Final Report: Grant Award DE FG52 03 SF22689 (formerly DE FG03 03 SF22689)

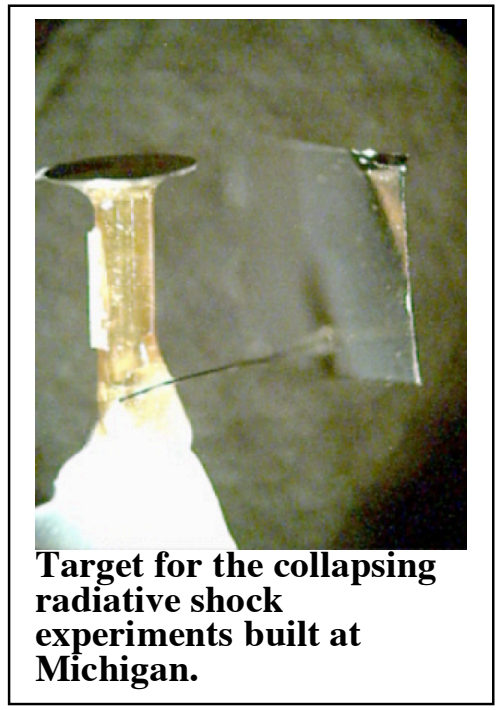

$$
\begin{gathered}
\text { Final Report } \\
\text { Principal Investigator / } \\
\text { Project Director: } \\
\text { Professor R. Paul Drake } \\
\text { Tel. 734-763-4072 } \\
\text { FAX 734-647-3083 } \\
\text { email rpdrake@umich.edu } \\
\text { Institution: } \\
\text { University of Michigan } \\
\text { 2455 Hayward St. } \\
\text { Ann Arbor, MI 48109 }
\end{gathered}
$$

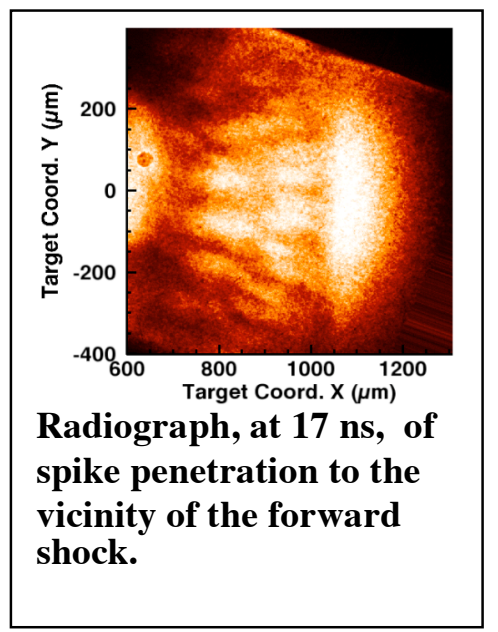

Title:

\title{
Experimental Astrophysics on the Omega Laser
}

Co Investigators:

\section{Bruce Remington \\ $L L N L$ \\ Harry Robey \\ $L L N L$}

Gail Glendinning

$L L N L$

Dimitri Ryutov

$L L N L$

Michel Koenig

LULI, Ecole Polytechnique

France

Aaron Miles

$L L N L$

Brent Blue

$L L N L$
Dave Arnett

University of Arizona

James Stone

Princeton University

Serge Bouquet

CEA, France

Mark Hermmann

LLNL

Yongmin Zhang

State University of New York.

Stony Brook

Andrew Mackinnon

$L L N L$

Freddy Hansen

$L L N L$
Robert Rosner

University of Chicago

Tomasz Plewa

University of Chicago

Jim Knauer

LLE; University of Rochester

Tom Boehly

LLE; University of Rochester

James Glimm

State University of New York-

Stony Brook

A project funded by the National Laser Users' Facility

National Nuclear Security Administration

Office of Defense Science

Albuquerque Service Center 
Final Report: Grant Award DE FG52 03 SF22689 (formerly DE FG03 03 SF22689)

\section{Introduction and motivation}

We provide here the following report on our activities. We have organized this report to discuss the motivation for our work, our experimental accomplishments, and other impact of our work.

It is just over ten years since the realization that one can use High-Energy-Density (HED) facilities to study dynamical processes that matter for astrophysics, ${ }^{3,4}$ complementing their established use to study material properties such as opacity ${ }^{6}$ and equation of state. ${ }^{7}$ This was not long after observations of SN 1987A showed that we do not understand the structure of supernovae (SNe). It was roughly coincident with SN 1993J, whose differences with SN 1987A (see Fig. 1) dramatized the importance of radiative shocks in some Type II supernovae. ${ }^{8}$ It was also not long after publication of simulations of the radiative phase that SN 1987A (and all supernova shocks) pass through. ${ }^{9}$ Our collaboration brings together motivated students, expert experimenters, senior theorists, and major simulation groups to use Omega to address aspects of these issues. We are now poised to observe the impact of realistic initial conditions on the structure that develops at an interface like that in an exploding star, and to study the scaling and structure of radiative shocks in a relevant regime.

In SN1987A, the observation of heavy elements from the core of the star, and evidence of the associated heating by radioactive decay, took place much sooner than was predicted by existing models of SN explosions. ${ }^{15,16}$ This showed conclusively that the structure of $\mathrm{SNe}$ was not understood. The explosion induced by core collapse in a Type II supernova may be globally symmetric ${ }^{17,18}$ or highly asymmetric, ${ }^{23,}{ }^{24}$ but in either case a blast wave is formed that blows apart the bulk of the star. This in turn produces hydrodynamic instabilities that transport interior material outward. Simulations of complete $\mathrm{SNe}$ explosions to date have been only in 2D, and hydrodynamic simulations in 3D have examined only isolated spikes of dense material.

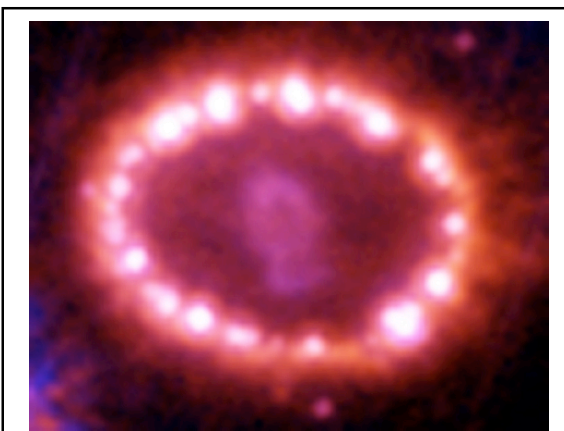

Figure 1. November 2003 image of SN 1987A. The dominant emission now is from the radiative shocks interacting with the ring of dense material that was present before the explosion.

Neither of these has produced enough transport of dense material to directly explain the observations of SN 1987A. In contrast, our NLUF experiments, with specific threedimensional initial conditions, show much larger transport than one would expect from such simulations. ${ }^{5}$ This is a dramatic and important result. These observations may well provide the key to resolving the two-decade-old problem of mix in supernovae. We have submitted a paper to Nature to make this point. ${ }^{26}$ But the only way to know whether these observations are the key to the SN mix problem is to carry out the program of research we are conducting. We are doing experiments to determine the causes of the larger transport, followed by experiments using initial conditions from new, state-of-the-art, three-dimensional simulations, by members of our team, of the progenitor star. The plan 
is to produce a definitive and well-understood result regarding the outward penetration of dense spikes of material in systems well scaled to exploding stars. We have also improved the experiments to obtain higher-quality data.

In a radiation hydrodynamic system, radiative fluxes or pressures are large enough to significantly affect the hydrodynamic evolution. Such systems are common in astrophysics and are more difficult to understand than purely hydrodynamic systems. For this reason, our astrophysical colleagues have consistently listed radiation hydrodynamics as a key area for HED laboratory astrophysics. Developing such experiments has not been easy, as they are an inherently more-challenging use of HED facilities, and as there has been much less preparatory work done in the context of inertial fusion. Our focus has been on the development of radiative shocks, which are shock waves from which the radiation is so intense that the flow of energy in the system is fundamentally altered. We have been working in a planar geometry to increase our ability to diagnose the shocks and to establish the potential to use them as sources. We have produced shock waves in this regime, have evaluated several design options for experiments and diagnostics, and have submitted a paper to Physical Review Letters $(P R L)$ based on this work. ${ }^{27}$ We are now ready to begin scaling experiments and to work to diagnose the structure of the shock in more detail, as is discussed further below. The ongoing scaling experiments should become a benchmark case for simulations of radiative hydrodynamic astrophysical systems.

Extensive student involvement has been a key an aspect of this project. During the project, 8 graduate students have made a total of 18 trips to our NLUF experiments, and 13 undergraduate students have made a total of 25 trips. During this period, one Ph.D. thesis has been based on simulations of the data obtained by this NLUF project. ${ }^{28}$ At Michigan, students are engaged in construction of targets, data analysis, experiment design, and computer simulations related to this project. There is substantial educational benefit from target construction and metrology by students; we have added this capability and have successfully built gas targets during the past two years. In addition, the students of our collaborators are conducting simulation studies related to the project.

Our team includes leaders in astrophysics, nonlinear hydrodynamics, and ICF to attack these problems of fundamental interest to astrophysics and to DOE. The participation of the ASC FLASH Center at the University of Chicago allows us to move beyond the "simple" experiments of the past toward experiments that test computational models at an unprecedented level of detail and complexity. Progress made utilizing state-of-the-art codes (at the University of Arizona, at Stony Brook, and at CEA (in France), the FLASH Center, and in the DOE labs) on our ICF-scale experiments should prove to be very useful to DOE/NNSA. The rigorous scaling under various conditions being developed as part of this work will also find utility in scaling from current Omega experiments to ignition experiments being planned for NIF.

We believe that our work through NLUF is the shining example of world-class academic HED research on the NNSA facilities. It has had a broad impact, both within the refereed literature and beyond. The 17 publications from this project during the past two years 
include the submissions to Nature and to PRL and published papers in PRL, Physics of Plasmas, the Astrophysical Journal and elsewhere. These are listed in the bibliography and referenced here. ${ }^{5,22,25-27,29-40}$ In addition, we have had an impact in other ways. Our work has produced numerous invited talks at scientific conferences, including one at the 2003 APS/DPP meeting, two at the associated miniconference on Laboratory Astrophysics, and one at the IPELS meeting in 2003, and two talks at the APS spring meeting in Albuquerque in 2002. The P.I. was also selected as a Distinguished Lecturer in Plasma Physics by the Division of Plasma Physics of the American Physical Society in 2003. Our work and our presentations to the panels played a role in the recommendations and reports of two NRC panels: the "Committee on the Physics of the Universe" and the "Committee on High Energy Density Plasma Physics". In addition, a BBC documentary entitled "Hyperspace, which includes a segment shot at Omega and featuring our work, is now available in bookstores.

Further evidence of our impact comes from Prof. Nomoto of the University of Tokyo, who is one of the world's leading theorists of supernova explosions. Following the mostrecent conference on High Energy Density Laboratory Astrophysics (HEDLA), he wrote to the PI: "I enjoyed very much HEDLA-2004 in Tucson. It was rather a surprise to see how the experimental approach has been actually successful. My impression in earlier meetings (already a few years ago) was that most of presentation were planning or numerical simulations, but this time I saw real experimental results, which was fun." Much of the data to which Dr. Nomoto refers was obtained on Omega by our collaboration, from which there were 7 presentations at the conference.

\section{Structure in Supernovae A. Importance of the work}

Human understanding of stellar explosions is in a period of significant advance. Improvements in instrumentation, in computer simulations, and in laboratory experiments all are contributing. One particular recent supernova, SN1987A, has played an important role because, at a distance of 150,000 light years, it is relatively close to Earth. SN 1987A was a core-collapse supernova -- the gravitational collapse of its iron core released the energy that drove the explosion. One process that occurs during such explosions is the Rayleigh-Taylor (RT) instability, which has the potential to transport some material from deep within the star to its outer layers. Our experiments under this grant show the transport of material much further than one would anticipate from existing theories and supernova simulations.

In SN1987A, the early observation of $\gamma$-rays from ${ }^{56} \mathrm{Co}$, relative to the timing anticipated from simulations assuming spherical symmetry, the shape of the $\mathrm{x}$-ray and visible light curves, and the high velocity of $\mathrm{Ar}, \mathrm{Ni}, \mathrm{Co}$, and other elements $(\sim 3000 \mathrm{~km} / \mathrm{s})$, provided compelling evidence that the explosion was not spherical. ${ }^{15,16,41}$ These observations could be explained by assuming that a small fraction of the heavy elements from the interior of the star had somehow been mixed into its outer layers. Furthermore, the shape of the xray spectrum implied that such mixing was non-uniform. ${ }^{16}$ Explaining these observations 
Final Report: Grant Award DE FG52 03 SF22689 (formerly DE FG03 03 SF22689)

in detail has proven to be an enduring challenge. Straightforward modeling of hydrodynamic instabilities has not produced enough mixing to explain them. ${ }^{17,42-47}$ Our past and ongoing experiments, in combination with simulations, should impact the consideration of instabilities as a potential explanation for the observations from SN1987A.

Two hypotheses that might explain the astrophysical observations have had a significant presence in the literature. First, the transition zones between regions in the star, across which the dominant element changes and the density drops more rapidly, become unstable to the Richtmeyer-Meshkov (RM) instability ${ }^{48,49}$ and then to the RayleighTaylor (RT) instability ${ }^{5,51}$ in response to the passage of the blast wave that blows the star apart. $^{52-54}$ The hypothesis is that these instabilities produce spikes of dense material that penetrate into the hydrogen layer of the exploding star. We emphasize that explaining the observations from SN1987A requires only that a tiny amount $(\sim 1 \%)$ of the ${ }^{56} \mathrm{Co}$ be accelerated to velocities above $3,000 \mathrm{~km} / \mathrm{s} .{ }^{15},{ }^{16}$ From spherically symmetric models one can conclude that mixing is necessary but can gain little insight regarding the mechanisms. ${ }^{55,56}$ In 2D models, one can observe the evolution of structure due to RT and RM. ${ }^{17,42-46}$ The amount of penetration turns out to be strongly sensitive to the initial conditions. Fryxell, Muller, and Arnett ${ }^{17,42,46}$ perturbed the unstable interfaces, but did this randomly rather than on a physical basis and also did not evaluate or include the structure produced by neutrino-driven convection. They found penetration of high-Z material out into the hydrogen layer, though not to high-enough velocity to explain the observations. In contrast, Kifonidis et al. modeled the perturbations produced by neutrino-driven convection but included no perturbations of the outer interfaces. ${ }^{18}$ They found that the spikes of high-Z material encountered a well established "reverse shock" at the He-H interface, which slowed them significantly. Spikes do experience less drag and reach higher velocities in $3 \mathrm{D}$ than in $2 \mathrm{D}$. However, there are no $3 \mathrm{D}$ simulations of $\mathrm{SN}$ explosions, and the velocities remain too small to explain SN 1987A in 3D simulations ${ }^{47}$, ${ }^{57-59}$ and buoyancy-drag models ${ }^{60,61}$ of isolated 3D spikes. Other existing studies of 3D RT instabilities, reviewed in Drake et al., ${ }^{5}$ are of limited relevance for reasons discussed there.

The second hypothesis that might explain the observations of SN 1987A is that the explosion might be driven by a jet from the core, which could also rapidly transport high$\mathrm{Z}$ material outward, at least near the poles (See Wang et al. and refs therein ${ }^{62}$ ). There is evidence that SN1987A and many other SN explosions are asymmetric, but the detailed mechanisms that might produce jet-driven explosions are not well understood. Note that in order to blow apart the bulk of the star, even in a jet-driven explosion, a blast wave must be driven through it. The blast wave is presumably the bow shock from the jet, but the details of jet-driven explosions are not understood and research in this direction has only been initiated [see, for example, ${ }^{23}$ Khokhlov et al.] Any such blast wave will produce RM and RT instabilities, and our work here contributes to the understanding of their impact in such models.

Experiments at high energy density now provide an independent option for the examination of hydrodynamic processes that may occur in astrophysical environments. 
We report here an experiment in the correct scaling regime, described by the same equations that describe the plasma in supernovae. As has been discussed in several papers, ${ }^{63-65}$ under such conditions the evolution of a laboratory system can be identical to an astrophysical one. This requires that the spatial structure and boundary conditions of the two systems be identical in an appropriately scaled sense and that the structure of the equation of state be the same. In practice, one can produce an experimental system whose evolution will parallel the astrophysical one over some period in time and space, beyond which the evolution of the experimental system is affected by its limited boundaries.

\section{B. Research under the existing NLUF award}

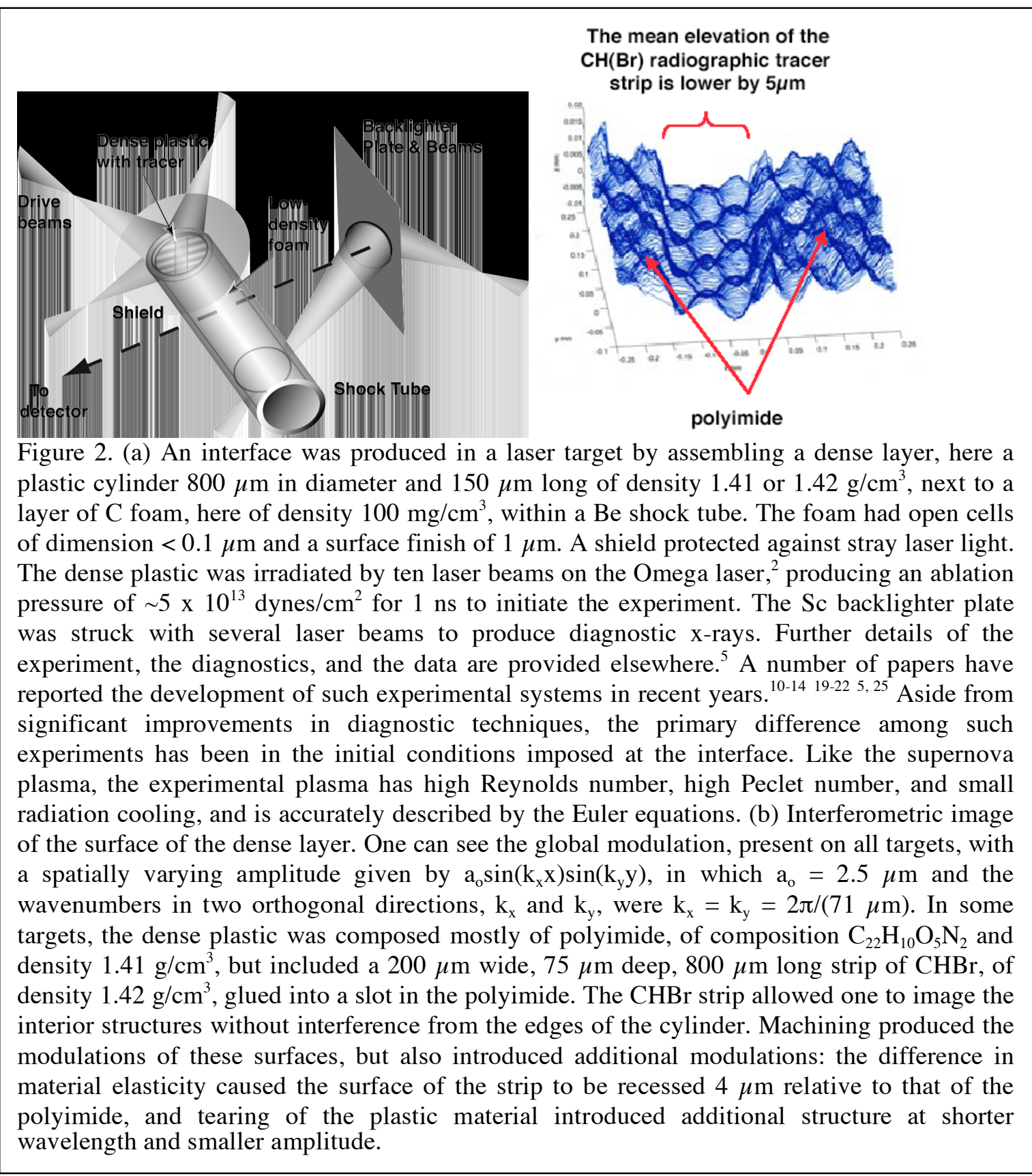


Results were obtained in the first such experiments to impose a 3D structure at an interface. Figure 2 shows the experimental system, and Figure 3 shows the 1D evolution of the interface. The present experiments compare the evolution of the interface from two similar initial conditions. In both cases, the surface of the dense layer was modulated in the eggcrate-like pattern seen throughout Fig. 2b. In some targets, the dense plastic was entirely $\mathrm{C}_{500} \mathrm{H}_{457} \mathrm{Br}_{43}(\mathrm{CHBr}$ below), which was cast against a smooth mold to produce a smoothly modulated surface. In other targets, machining of the interface produced both a large depressed region and additional small-scale structure.

Figure 3 shows the evolution of density structure in the experiment, from a simulation with the 1D, Lagrangian, single-fluid, three-temperature, radiation-hydrodynamic code HYADES with multigroup diffusive radiation transport. ${ }^{66}$ At 1 ns, pressure from laser ablation has driven a shock wave into a block of dense material. The pressure ends at $1 \mathrm{~ns}$, so that the rarefaction of the driving surface overtakes the shock, forming a blast wave, before the shock reaches the interface, as seen at $2 \mathrm{~ns}$. The structure at later

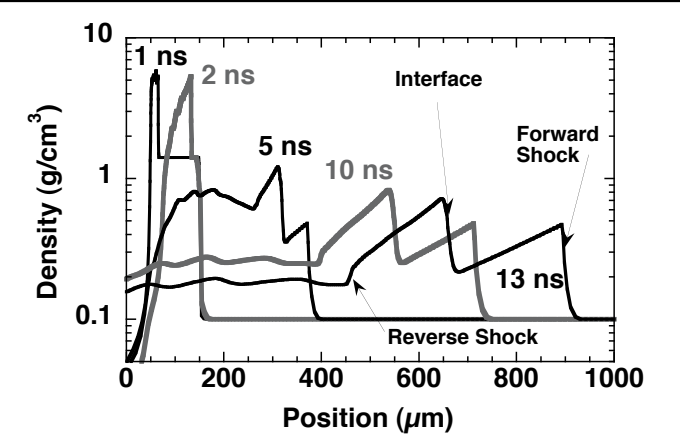

Figure 3. Evolution of density structure. times is then characteristic of an interface that has been processed by a blast wave, with a forward shock in the foam and a developing reverse shock in the plastic. The density drops roughly fivefold at the interface between the plastic and the foam. This ratio stays constant as the interface decelerates and the pressure decreases. This plasma is dense enough that the electron and ion temperatures equilibrate rapidly. The plasma parameters for the experiment and for the hydrogen-helium interface in SN 19878A confirm the validity of the scaling between the experiment and SN 1987A. ${ }^{63}$.

Figure 4 shows radiographic images from these experiments, and an additional image is shown on the cover. The more complex initial conditions produce spikes that extend much further and that appear to overtake the forward shock. These spikes are much longer that typical buoyancy-drag models would predict. ${ }^{5}$ The precise cause of this is the subject of ongoing research, but reasonable hypotheses include the introduction of additional vorticity to the spikes by the additional structures at the interface and increased interactions of the spikes due to the influence of the additional structures. It is quite clear, however, that initial conditions determine how far the spikes penetrate. [This has also been seen in hydrodynamic simulations of planar systems in two dimensions. ${ }^{25}$ ] These experiments show that penetration of the spikes to the region of the forward shock is possible. 

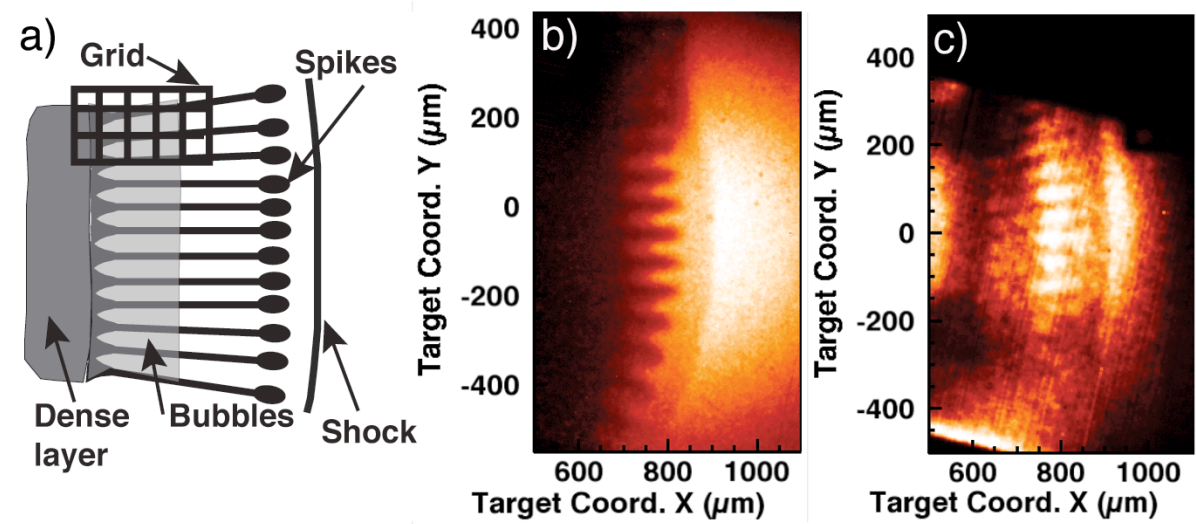

Figure 4. Radiographic images from these experiments, in which the diagnostic $x$-rays were primarily absorbed by $\mathrm{Br}$ in the dense plastic material, allowing one to observe the interface between the two materials. (a) A sketch showing the primary features seen in these images. (b) Image at $13 \mathrm{~ns}$ of a target with a smoothly modulated interface. The spikes remain well behind the forward shock. This type of target does not provide good viewing of the bubbles. (c) Image at $13 \mathrm{~ns}$ of a target with a more-structured interface. The spikes extend to the approximate location of the forward shock. Such spikes cannot penetrate readily through the forward shock, as the drag they experience greatly increases there.

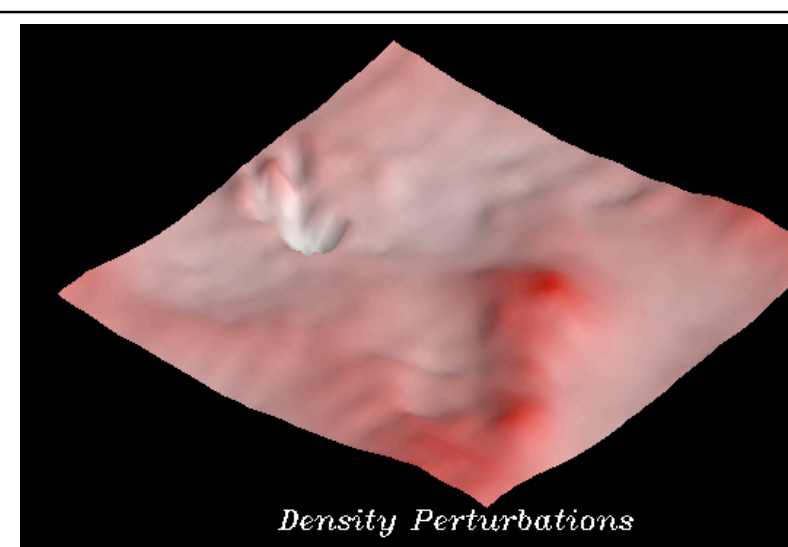

Figure 5. Density perturbations at the He-H composition interface. Shown here as a surface plot is density from a slice at fixed radius of a 3D computational wedge that is $\mathbf{1 5}$ degrees on a side. Simulations in 3D were necessary to accurately capture the scale of the convective perturbations. These simulations of convection used Prometheus, a piecewise-parabolic-method (Godunov-type) hydrodynamics code with diffusive radiative transfer and state-of-the-art stellar microphysics and nuclear reaction rates. The initial conditions for these calculations are a 23 solar mass model, approximately the mass of the SN1987A progenitor, which was evolved with TYCHO, a publicly available, one-dimensional, stellarevolution code developed at the University of Arizona by Dave Arnett and his students. The typical resolution is 300 radial zones and 40,000 angular zones.
Thus, one concludes that initial conditions are the critical issue that determines whether RT can explain SN 1987A data. While the initial conditions to which the stellar explosion is subject may seem like an impenetrable mystery, in fact researchers have begun to make progress in specifying them. It has become clear that the interfaces between shells in the presupernova star are structured. Convective burning shells in pre-core-collapse stellar models are unstable, and determine the structure of composition interfaces, as well as the associated spectrum and amplitudes of density perturbations at these interfaces. These 
perturbations will ultimately give rise to and shape instabilities in the passage of an outward moving supernova blast wave. The nature of these conditions is now being examined for the first time in threedimensional,

hydrodynamic stellar models. Figure 5 shows the distribution of

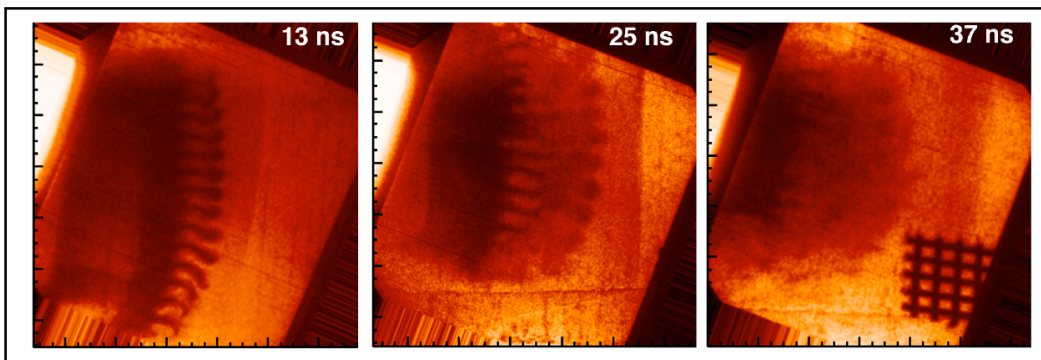

Figure 6. Radiographs using a backlit pinhole. The initial conditions of each target included a 2D sinusoid across the entire target having a $50 \mu \mathrm{m}$ wavelength and a $5 \mu \mathrm{m}$ full amplitudes. Half of each target had an additional mode with 5 $\mu \mathrm{m}$ wavelength and $0.5 \mu \mathrm{m}$ amplitude.

perturbations that arise at a composition interface at the outer edge of a convection zone in a supernova progenitor star. Note the presence of three-dimensional structure with local perturbations at a range of spatial scales.

\section{Recent developments}

The very exciting result just described, leading to a submission to Nature, suggests a sequence of further experiments now underway. These experiments employ improved diagnostics and specifically a backlit pinhole. In a backlit pinhole configuration, a small xray source is created on a target placed about $1 \mathrm{~cm}$ from the primary target (and positioned separately). This x-ray source illuminates (in order) a pinhole, the target, and a detector. We (and others) have demonstrated the use of this diagnostic during the past two years. It has better signal-to-noise than traditional area backlighting. Figure 6 below shows data from an experiment we did to demonstrate this technique. However, there is more risk associated with this technique than with traditional area backlighting. Small errors in alignment of the backlighter or in diagnostic timing can more easily cause the loss of data. A simulation of these experiments, by Aaron Miles, is shown in Fig. 7.

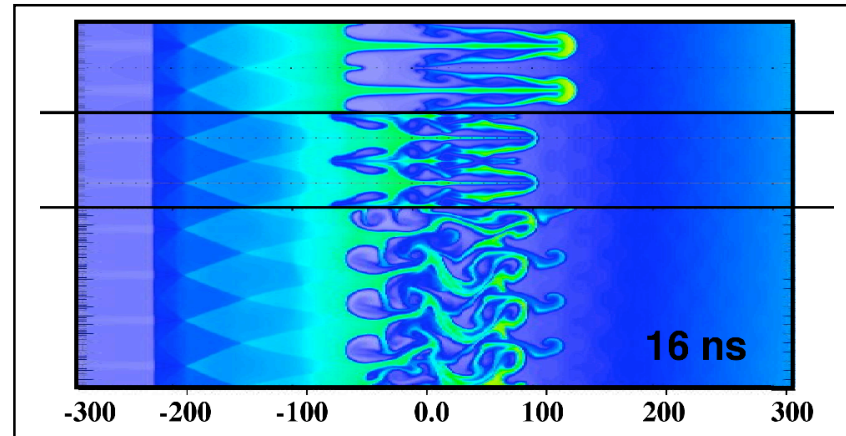

Figure 7. Density structure from a CALE simulation of the experiment of Fig. 6, with variations in initial conditions. The top shows a single mode, the middle shows two modes in phase, and the bottom shows two modes out of phase. 
Final Report: Grant Award DE FG52 03 SF22689 (formerly DE FG03 03 SF22689)

\section{Radiative Shocks A. Importance of the work}

Radiation plays an important role in many astrophysical shock waves. If the region upstream or downstream of the shock is optically thin, then the density of the shocked layer can increase greatly as it cools by radiating away its energy. ${ }^{35,67,68}$ The thickness of the shocked layer correspondingly decreases, so the shock can be said to collapse. The shock wave emerging from a supernova passes through regimes in which the shocked layer first radiates strongly enough to produce a radiative precursor and then collapses in space because of radiative energy losses. ${ }^{69}$ Similar dynamics can occur at the accretion shocks produced during star formation, ${ }^{70-72}$ and at the reverse shock in a supernova remnant formed from a star with a dense stellar wind ${ }^{73}$ or pre-existing dense material as in SN 1987A. ${ }^{74}, 75$ There is more generally a radiative cooling zone behind most astrophysical shocks. Collapse of an existing shocked layer can occur in some cases, as for example in aging supernova remnants in which the shocked layer is no longer driven. ${ }^{76}$ The production and study of laboratory shock waves in which radiation matters is relatively new. In this letter we report the production and observation of a collapsing radiative shock in planar geometry.

The thin, dense layers that are produced when shocked material collapses are subject to hydrodynamic instabilities like those discussed by Vishniac and Ryu. ${ }^{77}$, 78 These instabilities produce convolutions in the dense layer, observed in simulations ${ }^{69,76}$ and believed to be responsible, for example, for the structure of the ring nebula in Wolf-Rayet stars. $^{79}$ The experimental system described here produces an isolated, dense layer that may permit the observation of the early phases of such instabilities in well-understood conditions.

One can organize the prior work on radiating shock waves by the strength of the radiative effects that were present. Two experiments have produced radiative effects at velocities low enough that radiation produced in consequence of shock heating is not the dominant effect. The experiments of Grun et al.$^{80}$ appear to have produced the Vishniac instability, by driving a blast wave through xenon gas under conditions that produce strong radiation from low ionization states of xenon during the shock transition. ${ }^{81}$ In the experiments discussed $^{82}$ by Edwards et al., radiation from a shocked layer that was further heated by electron heat conduction affected the medium upstream of the shock. The threshold ${ }^{29}$ for the formation of a radiative precursor, through the action of the shock itself, is reached when the number of ionizing photons radiated by the shock exceeds the number of atoms approaching the shock. Radiative precursors have been observed in experiments by Bozier et al., Keiter et al., and Koenig et al. ${ }^{29,83,84}$

The present experiment is the first in planar geometry to exceed the threshold for radiative collapse through the formation of a post-shock cooling layer, and to detect the material that has been shocked and cooled. A post-shock cooling layer must form when the thermal radiative losses from the shocked material exceed the energy flux entering the shocked material. The approximate threshold for this can be expressed as $R_{r}>1$, where 
Final Report: Grant Award DE FG52 03 SF22689 (formerly DE FG03 03 SF22689)

$$
R_{r}=\frac{8}{\gamma(\gamma+1)} \frac{\sigma T_{i}^{4}}{\rho_{o} c_{v} T_{i} u_{s}}=\frac{8 \sqrt{2}}{\gamma(\gamma+1)^{2}} \frac{\sigma T_{i}^{5 / 2}}{\rho_{o} c_{v}^{3 / 2}}=\frac{64}{\gamma(\gamma+1)^{7}} \frac{\sigma}{\rho_{o} c_{v}^{4}} u_{s}^{5},
$$

in which $u_{s}$ is the shock velocity, $\sigma$ is the Stefan-Boltzmann constant, $\rho_{o}$ is the mass density of the unshocked, upstream material, $c_{v}$ is the specific heat at constant volume of the post-shock material, $\gamma$ is the polytropic index appropriate to the shock transition. The initial post-shock temperature is $T_{i}$, which assumes that the ions and electrons rapidly equilibrate behind the shock, as is the case in most laboratory experiments. The second two equalities exploit the strong-shock relation for the post-shock temperature,

$$
T_{i}=\frac{2}{(\gamma+1)^{2}} \frac{u_{s}^{2}}{c_{v}}=\frac{2}{(\gamma+1)^{2}} \frac{(\gamma-1) A m_{p}}{k_{B}(Z+1)} u_{s}^{2},
$$

in which $k_{B}$ is the Boltzmann constant, $A$ is the atomic weight of the upstream material, $m_{p}$ is the proton mass, and $Z$ is the average post-shock ionization. The second equality here serves to define $c_{v}$. Note that $c_{v}$ and $\gamma$ both should include the effects of ionization, especially in a material like xenon. The corresponding threshold velocity ${ }^{35}$ in xenon at $10 \mathrm{mg} / \mathrm{cm}^{3}$, is about $50 \mathrm{~km} / \mathrm{s}$. If the optical depth of the downstream and upstream regions is not comparable, then $R_{r}$ should be multiplied by the ratio of downstream to upstream optical depth, to account for the finite emissivity and absorptivity of these two layers.

\section{B. Research under the existing NLUF award}

We drive a planar, radiative shock through a xenon-filled target and observe the structure of the shocked xenon layer. Figure 9 shows a schematic of the targets. The inside diameter (ID) of the gas cell was either $600 \mu \mathrm{m}$ or $912 \mu \mathrm{m}$. We used the fill tube to evacuate the targets and fill them with xenon, producing a xenon pressure of approximately 1.1 ATM $\left(\rho=6 \mathrm{mg} / \mathrm{cm}^{3}\right)$ at the time of the experiment. The drive disks were $51 \mu \mathrm{m}$ of polyimide $( \pm 3 \%$ and at $\left.1.41 \mathrm{~g} / \mathrm{cm}^{3}\right)$, overcoated with $20(+5 /-$ 10) $\mu \mathrm{m}$ of polyvinyl at $1 \mathrm{~g} / \mathrm{cm}^{3}$, or 40 $\mu \mathrm{m}$ or $50 \mu \mathrm{m}$ thick Be $( \pm 7 \%)$. We focused ten laser beams of wavelength $0.35 \mu \mathrm{m}$ onto $\mathrm{a} \sim 2.5 \mathrm{~mm}$ diameter drive disk in a square, 1-ns flat-top pulse, with the midpoint of the rising edge defining time $t=0$, and a total energy up to 4000 J. Distributed Phase Plates (DPPs) created super-Gaussian focal spots of 720 or $820 \mu \mathrm{m}$ diameter (FWHM), with small scale structure superimposed. On some shots, using Smoothing by Spectral Dispersion (SSD) smoothed the beams further. The corresponding laser irradiance

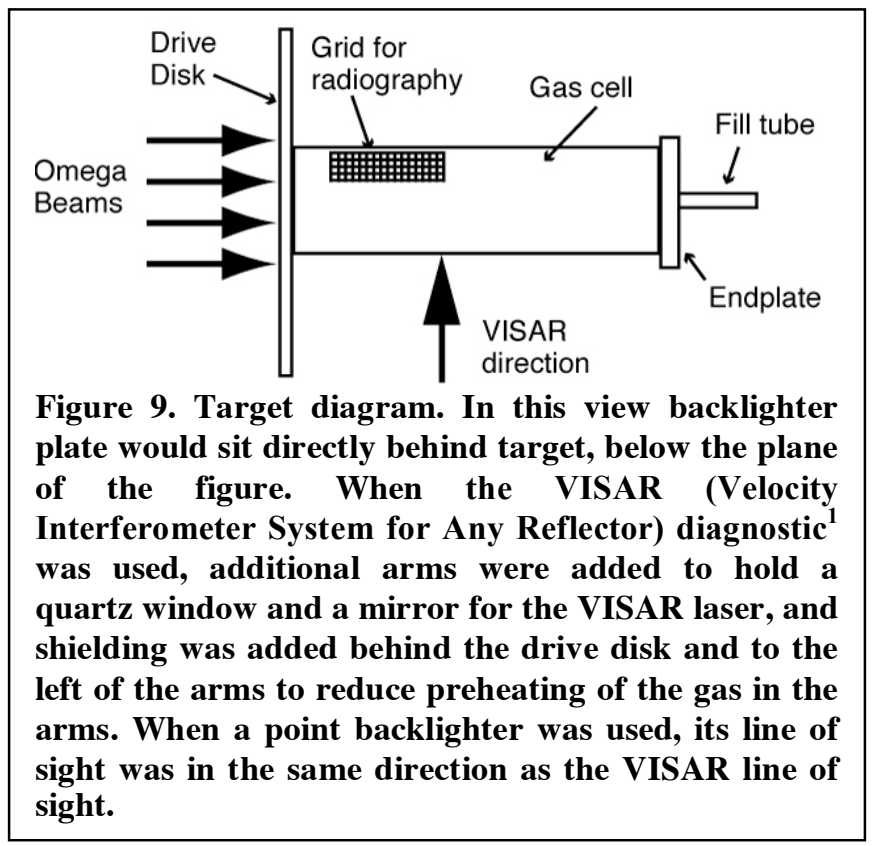




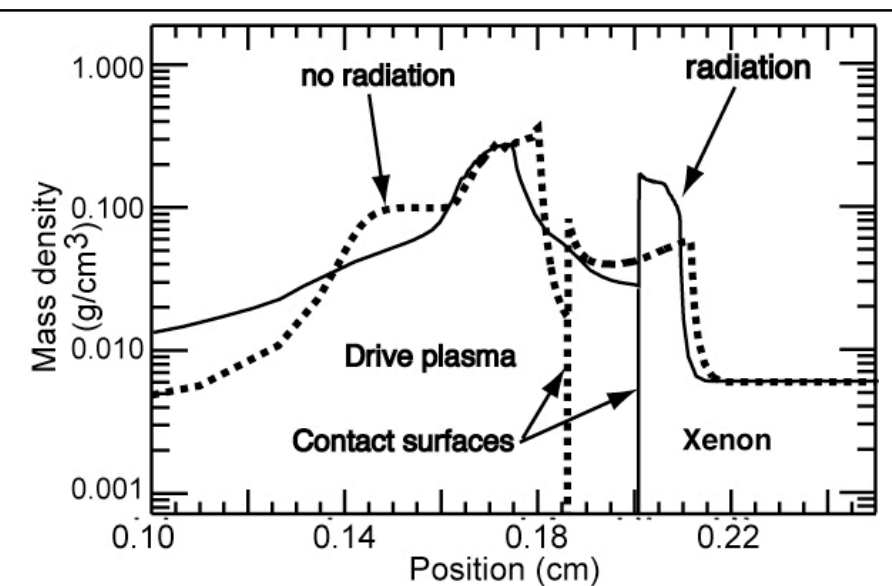

Figure 10. Density vs position from two simulations, at 18 ns, for experiments at a laser intensity of $8.5 \times 10^{14}$ $\mathrm{W} / \mathrm{cm}^{2}$. The boundaries between the drive plasma, in this case Be, and the xenon are shown, labeled "contact surfaces." The solid line shows the result for multigroup, diffusive radiation transport, while the dashed line shows the result for a non-radiative system. When radiation is suppressed (dashed line), a collapsed layer does not form. was up to $10^{15} \mathrm{~W} / \mathrm{cm}^{2}$. The pressure from laser ablation shocked and then accelerated the Be disk, launching it into the xenon and thereby driving a shock.

X-ray radiography was the principal diagnostic, using $\mathrm{x}$-ray framing cameras 85 as the detectors. The laser beams producing the x-rays were of the same wavelength and laser pulse as the above, at a nominal energy of $450 \mathrm{~J} /$ beam, without SSD and usually without DPPs. Each shot included an "area backlighter", in which such laser beams were focused to $\mathrm{a} \sim 1 \mathrm{~mm}$ spot on a foil several square $\mathrm{mm}$ in area and 5

$\mu \mathrm{m}$ thick. The foil was either Ti, with K-shell emission at $\sim 5.3 \mathrm{keV}$, or Sn, with L-shell emission from 3.5 to $3.9 \mathrm{keV}$. A "backlit pinhole" was also used on some shots. The laser beams were focused to a $300 \mu \mathrm{m}$ spot on a $5 \mu \mathrm{m}$ thick Ti foil, spaced by $100 \mu \mathrm{m}$ of $\mathrm{CH}$ behind a $50 \mu \mathrm{m}$ thick Ta substrate with a $25 \mu \mathrm{m}$ through hole, covered by another 100 $\mu \mathrm{m}$ of $\mathrm{CH}$. This object was located approximately $12 \mathrm{~mm}$ from the target, with the detector approximately $229 \mathrm{~mm}$ on the other side of the target. Because of vignetting, the effective source size for this measurement was $\sim 15 \mu \mathrm{m}$. A gold grid was placed on the target as a spatial indicator for radiography. Squares on the gold grid are $63 \mu \mathrm{m}$ center to center, which allows position measurements and places a spatial scale on each image. As is described in the caption to Fig. 9, VISAR was also used in some cases.

One can gain some insight into the impact of radiation on the experiment through simulations. Here we discuss the results of simulations of this system using the 1D, Lagrangian code HYADES. ${ }^{6}$ HYADES was run using multigroup, diffusive radiation transport with 90 photon groups, adjusted to resolve the edges in the xenon opacity at up to $6 \mathrm{keV}$. The equation of state of xenon was the SESAME table. In the regime of this experiment, the polytropic index $(\gamma)$ inferred from the table is in the range of 1.2 to 1.3 , as is appropriate for an ionizing medium that is dense enough that collisional recombination

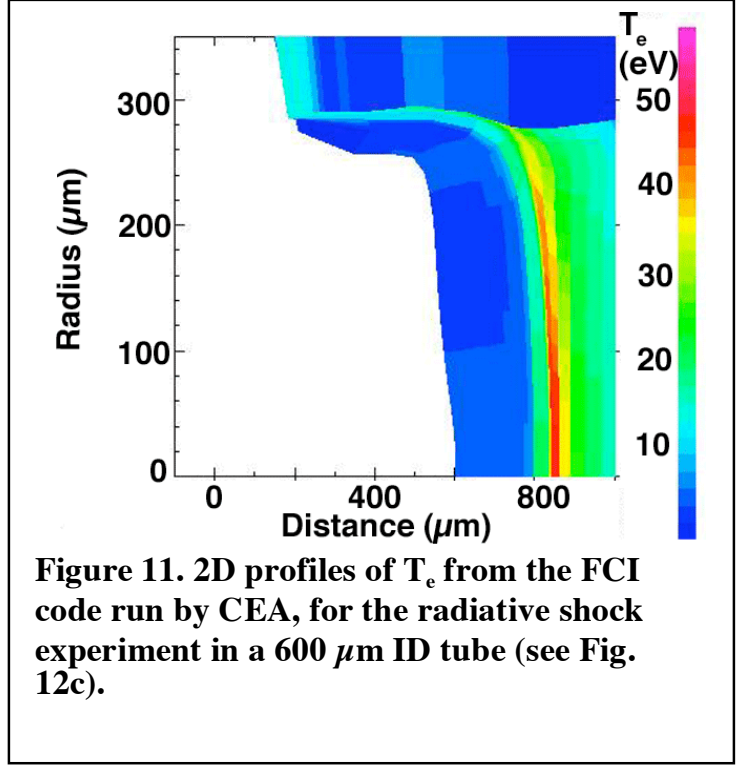


is dominant ${ }^{86}$ It is worth noting that the effective $\gamma$ of xenon can be significantly smaller in lower-density media in (more or less) coronal equilibrium, which is the case for the experiments with blast waves in gases. ${ }^{81,87}$ The xenon was modeled using an averageatom, LTE description, which one would expect to be only qualitatively accurate. The laser irradiance used in such 1D simulations must be reduced to give accurate results, because radial heat transport reduces the ablation pressure. ${ }^{88}$ Here it was adjusted to the level required to match the behavior of hydrodynamic experiments at similar laser intensity.

The solid curve in Figure 10 shows the radiative simulation results, while the dashed curve shows results of a simulation in which radiation is artificially suppressed. The shock transition is the rightmost increase in density, which is moving to the right into unshocked matter. The xenon layer is just to the left of the shock transition. In the radiative case one can see the post-shock density increase due to cooling. To the left of the contact surface is a more-structured layer of beryllium material. The structure in this layer has been established during the laser pulse, when there is shock reverberation in the driving material. The radiation has two effects. First, it narrows the shocked xenon layer by increasing its density. This is the primary effect one can detect using radiography. Second, it heats the beryllium that is driving the shock and causes the xenon layer to separate from the more-dense beryllium. As the layer radiates, radiation-driven ablation creates a distinct, low-density region between the dense driver material and the dense collapsed layer. ${ }^{89}$ This produces conditions favorable to the Vishniac instability discussed above.

To assess the role of two-dimensional effects in these experiments, our collaborators Laurent Boireau and Serge Bouquet of CEA Bruyeres ran 2D simulations using the code FCI, a Lagrangian, one-fluid-three-temperature code with multigroup diffusive radiation transport, an average atom LTE treatment of the materials, and flux-limited electron heat transport. Figure 11 shows the calculated profile of electron temperature, at $7 \mathrm{~ns}$, for a target with an initial gas density of $3 \mathrm{mg} / \mathrm{cm}^{3}$. One can see that the shock remains quite planar at this time, in this case with a $600 \mu \mathrm{m}$ gas cell and an $820 \mu \mathrm{m}$ laser spot. The density in the shocked layer at this time is 80 times the initial density, and there has been very little radial flow of mass out of the shocked layer. The decrease of temperature ahead of the shock is more rapid than in the 1D simulations, as one would expect with the inclusion of radial radiation losses.

Radiography of the shock in xenon shows clear indications of a thin, dense shock. In Figure 12a, one can see the center of the shock (which is moving to the right) at approximately $1600 \mu \mathrm{m}$. The region of highest opacity is narrow, being $45 \mu \mathrm{m}$ thick in this case. The backlit pinhole also obtained a less complete image on this shot, at $10.3 \mathrm{~ns}$, showing that the average shock velocity over this interval is $110 \mathrm{~km} / \mathrm{s}$. The velocity and position of the center of the shock are in this case within $10 \%$ of the values obtained from relevant 1D simulations, although in other experiments the agreement was not as close. The shock front is curved, and there are indications of a trailing layer of dense xenon along the wall of the tube. A similar experiment using a $40 \mu \mathrm{m}$ thick Be drive disk produced the backlit pinhole data of Fig. 12b. The bright feature to the lower right is part 

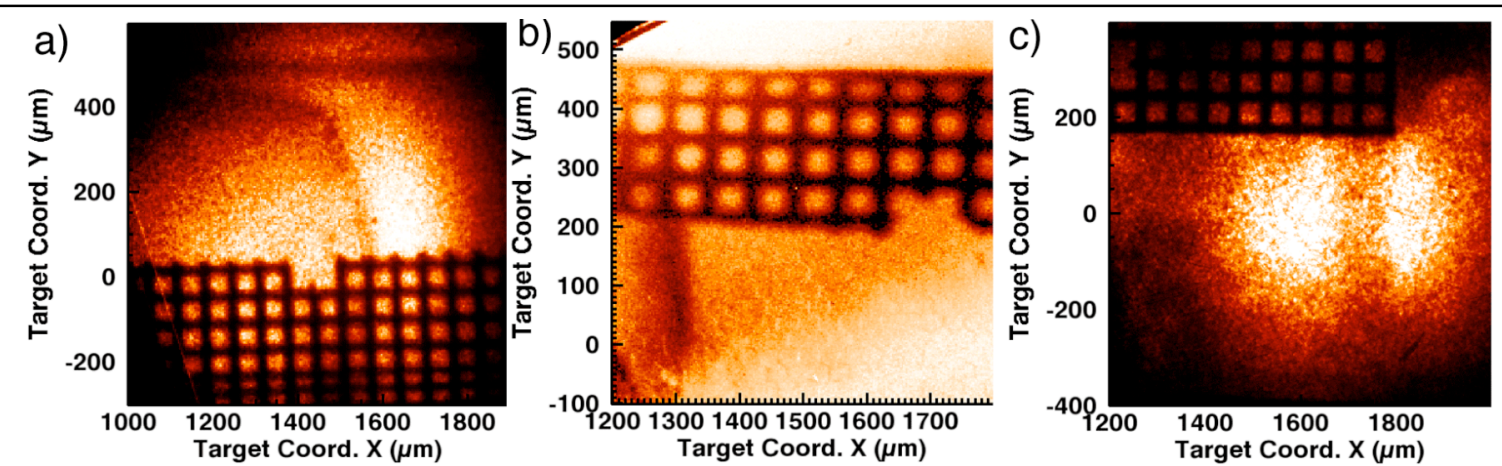

Figure 12. Radiography images from gas target experiments. (a) Area backlighting image, at 13.5 ns. (b) Image from backlit pinhole image, at $12.5 \mathrm{~ns}$. (c) Area backlighting image of smallerdiameter target $(600 \mu \mathrm{m}$ vs. $912 \mu \mathrm{m})$, at $18 \mathrm{~ns}$, using L-shell backlighter.

of a noise strip this instrument exhibited on this particular day; we expect much better data in future experiments. An experiment using a $600 \mu \mathrm{m}$ ID gas cell, an $820 \mu \mathrm{m}$ FWHM laser spot, no SSD, and a drive irradiance of $4.8 \times 10^{14} \mathrm{~W} / \mathrm{cm}^{2}$, diagnosed with Lshell x-rays from Sn at $18 \mathrm{~ns}$, showed a shock front that was much more planar and that had moved at $\sim 100 \mathrm{~km} / \mathrm{s}$ to that point, where its thickness was $80 \mu \mathrm{m}$, as Fig. 12c shows.

Fringe patterns from the VISAR diagnostic ceased before the drive laser shut off, as can be seen in Figure 13. Based on calculations, we attribute this to collisional absorption in the $\mathrm{Xe}$ gas, heated to a few eV by radiative preheat. At a late time, a thin feature which showed no fringes and which was non-uniform in space and duration was seen. We attribute this signal to reflection of the interferometer beam off from the edge of the shock front. The inferred shock velocity, based on this interpretation, is consistent with that determined from the radiographic data. Our collaborators from Ecole Polytechnique participated strongly in this aspect of the work, contributing to the interpretation of the data and sending a graduate student to the experiment.

The data of Fig. 12 clearly show a very thin shocked layer. The layer of xenon produced by a nonradiative shock, with an effective $\gamma$ of 1.2 to 1.3 , would be 140 to $220 \mu \mathrm{m}$ thick at the location seen, and the observed layer is $45 \mu \mathrm{m}$. Thus, one might suggest that the density has increased another factor of 3 to 4 in consequence of radiative losses, reaching a total of 34 times the initial xenon density. On the one hand, the inferred density increase would be reduced to whatever extent material has left the shocked region by flowing radially, although the $2 \mathrm{D}$ simulations find this to be small. On the other hand, this line of sight measurement will have a strong tendency to over-estimate the thickness of the shocked layer, due to any tilt, curvature, or rippling of the shock front. On balance, it appears reasonable

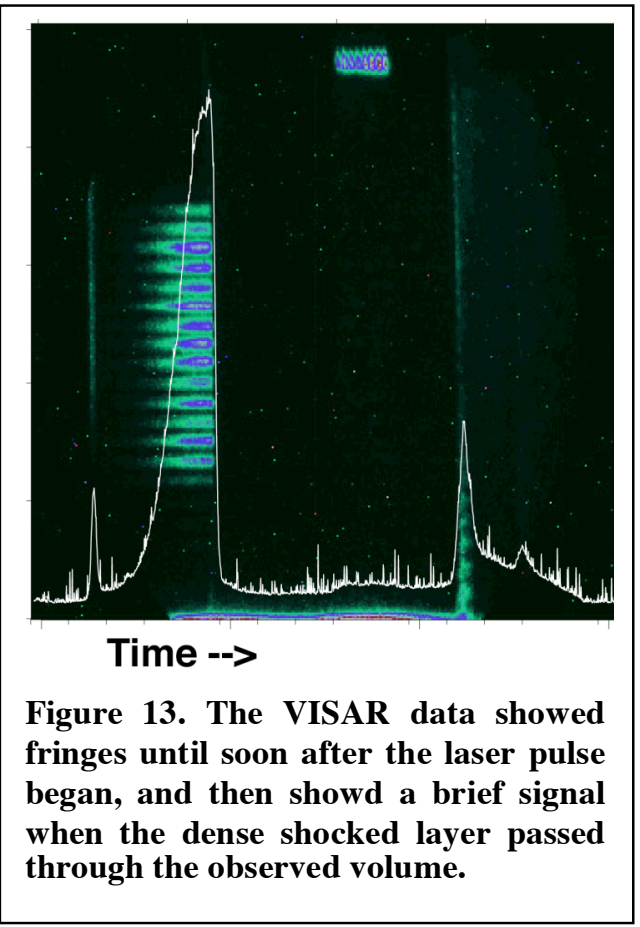


Final Report: Grant Award DE FG52 03 SF22689 (formerly DE FG03 03 SF22689)

to conclude that we have observed a thin layer of shocked xenon whose density has been increased significantly by radiative losses.

We also have used the 2D version of HYADES and the 2D, astrophysical code ZEUS to simulate this experiment at Michigan, but not yet satisfactorily. This led to a publication reporting the methodological developments that were necessary to achieve a successful simulation. ${ }^{90}$ 
Final Report: Grant Award DE FG52 03 SF22689 (formerly DE FG03 03 SF22689)

\section{BIBLIOGRAPHY}

1. Barker, L. M. \& Hollenback, R. E. VISAR. J. Appl. Phys. 43, 1669 (1972).

2. Boehly, T. R. et al. Initial performance results of the OMEGA laser system. Optics-Communications 133, 495-506 (1997).

3. Takabe, H. ICF and Supernova explosions. Jpn. Plasma Fusion Res. 69, 12851300 (1993).

4. Remington, B. A., Arnett, D., Drake, R. P. \& Takabe, H. Modeling astrophysical phenomena in the laboratory with intense lasers. Science 284, 1488-1493 (1999).

5. Drake, R. P. et al. Nonlinear mixing behavior of the three-dimensional RayleighTaylor instability at a decelerating interface. Phys. Plasmas 11, 2829-2837 (2004).

6. Springer, P. T. et al. Spectroscopic absorption measurements of an iron plasma. Phys. Rev. Lett. 69, 3735-3738 (1992).

7. Nellis, W. J., Ross, M. \& Holmes, N. C. Temperature measurements of shockcompressed liquid hydrogen: implications for the interior of Jupiter. Science 269, 1249-52 (1995).

8. Chevalier, R. A. Type II supernovae SN 1987A and SN 1993J. Science 276, 1374-1378 (1997).

9. Ensman, L. \& Burrows, A. Shock breakout in SN 1987A. ApJ 393, 742-755 (1992).

10. Kane, J. et al. Supernova-relevant hydrodynamic instability experiments on the Nova laser. Ap. J. 478, L75-L78 (1997).

11. Kane, J. et al. in Second Oak Ridge Symposium on Atomic and Nuclear Astrophysics (Oak Ridge, Tenessee, 1998).

12. Kane, J. et al. Scaling supernova hydrodynamics to the laboratory. Physics of Plasmas 6, 2065-2072 (1999).

13. Kane, J. et al. Supernova experiments on the Nova laser. Ap. J. Suppl. 127, 365369 (2000).

14. Kane, J. O. et al. Interface imprinting by a rippled shock using an intense laser. Phys. Rev. E 63, 055401R (2001).

15. Arnett, W. D., Bahcall, J. N., Kirschner, R. P. \& Woolsey, S. E. Supernova 1987A. Ann. Rev. Astron. Astrophys. 27, 629-700 (1989).

16. Chevalier, R. A. Supernova 1987A at five years of age. Nature 355, 691-6 (1992).

17. Fryxell, B., Muller, E. \& Arnett, D. Instabilities and Clumping in SN 1987A. I. Early Evolution in Two Dimensions. Ap. J. 367, 619-34 (1991).

18. Kifonidis, K., Plewa, T., Janka, H.-T. \& Muller, E. Nucleosynthesis and clump formation in a core-collapse supernova. ApJ Lett 531, L123-126 (2000).

19. Remington, B. A. et al. Supernova hydrodynamics experiments on the Nova laser. Phys. Plasmas 4, 1994-2003 (1997).

20. Remington, B. A. et al. in Supernova 1987A: Ten Years After in press (Chile, 1997).

21. Robey, H. F. et al. An experimental testbed for the study of hydrodynamic issues in supernovae. Phys. Plasmas 8, 2446-2453 (2001).

22. Robey, H. F. et al. The onset of turbulence in high Reynolds number, accelerated flows. Part II. Experiment. Phys. Plasmas 10, 614 (2003). 
Final Report: Grant Award DE FG52 03 SF22689 (formerly DE FG03 03 SF22689)

23. Khokhlov, A. M., Hoflich, P. F., Oran, E. S., Wheeler, J. C. \& Wang, L. Jetinduced explosions of core collapse supernovae. ApJ Lett. 524, L107-110 (1999).

24. Wang, L. et al. The axisymmetric ejecta of supernova 1987A. ApJ 579, 671-677 (2002).

25. Miles, A. R., Edwards, M. J. \& Robey, H. F. in Inertial Fusion and Science Applications submitted (Monterey, CA, 2003).

26. Drake, R. P. et al. Rayleigh Taylor spike penetration in core collapse supernovae. Science, in preparation (2004).

27. Reighard, A. B. et al. Collapsing Radiative Shocks in Xenon on the Omega Laser. Phys. Rev. Lett., submitted (2004).

28. Miles, A. in Physics (University of Maryland, College Park, 2004).

29. Keiter, P. A. et al. Observation of a hydrodynamically-driven, radiative-precursor shock. Phys. Rev. Lett. 89, 165003/1-4 (2002).

30. Calder, A. et al. On validating an astrophysical simulation code. Astrophys. J. 143, 201-229 (2002).

31. Robey, H. F. The effects of viscosity and mass diffusion in hydrodynamically unstable plasma flows. Phys. Plasmas, in press (2004).

32. Miles, A. R. et al. Numerical simulation of supernova-relevant laser-driven hydrodynamics experiments on Omega. Phys. Plasmas, in press (2004).

33. Miles, A. R. et al. The effect of a short wavelength mode on the evolution of a long wavelength perturbation driven by a strong blast wave. Phys. Plasmas, submitted (2004).

34. Drake, R. P., Remington, B. A. \& Ryutov, D. D. Experimental Astrophysics with Intense Lasers. Rev. Mod. Phys., submitted (2003).

35. Drake, R. P. Radiative Shocks in Astrophysics and the Laboratory. Astrophysics and Space Science, submitted (2004).

36. Danneberg, K. K. et al. in Inertial Fusion and Science Applications in press (Monterey CA, 2003).

37. Reighard, A. B. et al. in Inertial Fusion and Science Applications (American Nuclear Society, Monterey CA, 2003).

38. Leibrandt, D. R., Drake, R. P. \& Stone, J. M. ZEUS-2D Simulations of LaserDriven Radiative Shock Experiments. Astrophysics and Space Science, submitted (2004).

39. Kuranz, C. C. et al. Preheat issues in hydrodynamic HEDLA experiments. Astrophysics and Space Science, submitted (2004).

40. Kuranz, C. C. et al. Progress toward the study of laboratory scale, astrophysically relevant, turbulent plasmas. Astrophysics and Space Science, submitted (2004).

41. Hillebrandt, W. \& Höflich, P. The supernova 1987A in the Large Magellanic Cloud. Rep. Prog. Phys. 52, 1421-1473 (1989).

42. Arnett, D., Fryxell, B. \& Muller, E. Instabilities and nonradial motion in SN 1987A. ApJ Lett. 341, 63-6 (1989).

43. Hachisu, I., Matsuda, T., Nomoto, K. \& Shigeyama, T. Nonlinear growth of Rayleigh-Taylor instabilities and mixing in SN 1987A. Ap. J. Lett. 358, L57-61 (1990). 
Final Report: Grant Award DE FG52 03 SF22689 (formerly DE FG03 03 SF22689)

44. Herant, M. \& Benz, W. Hydrodynamical instabilities and mixing in SN 1987A: two-dimensional simulations of the first three months. ApJ 370, L81-84 + plate (1991).

45. Herant, M., Benz, W. \& Colgate, S. Postcollapse hydrodynamics of SN 1987A: two-dimensional simulations of the early evolution. Ap. J. 395, 642-653 (1992).

46. Muller, E., Fryxell, B. \& Arnett, D. Instabilities and Clumping in SN 1987A. A\&A 251, 505-514 (1991).

47. Kane, J. et al. Two-dimensional versus three-dimensional supernova hydrodynamic instability growth. Ap. J. 528, 989-994 (2000).

48. Richtmyer, R. D. Taylor instability in shock acceleration of compressible fluids. Commun. Pure. Appl. Math 13, 297 (1960).

49. Meshkov, E. E. Instability of the Interface of Two Gases Accelerated by a Shockwave. Sov. Fluid Dyn. 4, 101 (1969).

50. Rayleigh, L. Scientific Papers II (Cambridge, Cambridge, England, 1900).

51. Taylor, S. G. Hydrodynamic Instabilities. Proc. R. Soc. A201, 192 (1950).

52. Falk, S. W. \& Arnett, W. D. A Theoretical Model for Type II Supernovae. ApJ 180, L65 (1973).

53. Chevalier, R. A. The hydrodynamics of Type II supernovae. ApJ 207, 872-877 (1976).

54. Bandiera, R. Convective Supernovae. A\&A 139, 368-374 (1984).

55. Shigeyama, T., Nomoto, K. \& Hashimoto, M. Hydrodynamical models and the light curve of supernova 1987A in the Large Magellanic Cloud. A\&A 196, 141151 (1988).

56. Shigeyama, T. \& Nomoto, K. Theoretical light curve of SN 1987A and mixing of hydrogen and nickel in the ejecta. Ap. J. 360, 242-256 (1990).

57. Tryggvason, G. \& Unverdi, S. O. Computations of three-dimensional RayleighTaylor instability. Phys. Fluids A 2, 656-659 (1990).

58. Dahlburg, J. P., Gardner, J. H., Doolen, G. D. \& Haan, S. W. The effect of shape in the three-dimensional ablative Rayleigh-Taylor instability. I. Single-mode perturbations. Phys. Fluids B 5, 571-584 (1993).

59. Hecht, J. et al. Three-dimensional simulations and analysis of the nonlinear stage of the Rayleigh-Taylor instability. Laser and Particle Beams 13, 423-40 (1995).

60. Oron, D. et al. Dimensionality dependence of the Rayleigh-Taylor and Richtmyer-Meshkov instability late-time scaling laws. Phys. Plasmas 8, 28832890 (2001).

61. Dimonte, G. Spanwise homogeneous buoyancy-drag model for Rayleigh-Taylor mixing and experimental evaluation. Phys. Plasmas 7, 2255-2269 (2000).

62. Wang, L. et al. The axisymmetric ejecta of supernova 1987A. ApJ 579, 671-677 (2002).

63. Ryutov, D. D. et al. Similarity Criteria for the Laboratory Simulation of Supernova Hydrodynamics. Ap. J. 518, 821 (1999).

64. Ryutov, D. D., Drake, R. P. \& Remington, B. A. Criteria for scaled laboratory simulations of astrophysical MHD phenomena. Ap. J. Suppl. 127, 465-8 (2000).

65. Ryutov, D. D., Remington, B. A., Robey, H. F. \& Drake, R. P. Magnetohydrodynamic scaling: from astrophysics to the laboratory. Phys. Plasmas 8, 1804-1816 (2001). 
Final Report: Grant Award DE FG52 03 SF22689 (formerly DE FG03 03 SF22689)

66. Larsen, J. T. \& Lane, S. M. HYADES: a plasma hydrodynamics code for dense plasma studies. J. Quant. Spectrosc. Radiat. Transfer 51, 179-186 (1994).

67. Shu, F. H. The Physics of Astrophysics: Gas Dynamics (University Science Books, Mill Valley, CA, 1992).

68. Drake, R. P. \& Reighard, A. B. Structure of cooling layers in radiative shocks. in preparation (2004).

69. Ensman, L. \& Burrows, A. Shock Breakout in SN 1987A. Astrophysical Journal 393, 742-755 (1992).

70. Calvet, N. \& Gullbring, E. The Structure and Emission of the Accretion Shock in T Tauri Stars. ApJ 509, 802-818 (1998).

71. Hujeirat, A. \& Papaloizou, J. C. B. Shock formation in accretion columns-a 2D radiative MHD approach. A\&A 340, 593-604 (1998).

72. Lamzin, S. A. The structure of the shock wave in the case of an accretion onto low mass young stars. Astrophysics and Space Science 261, 137-140 (1999).

73. Chevalier, R. A. \& Fransson, C. Emission from circumstellar interaction in normal type II supernovae. Ap. J. 420, 268-285 (1994).

74. Borkowski, K. J., Blondin, J. M. \& McCray, R. X-rays and ultraviolet line emission from SNR1987A. Ap. J. 476, L31-L34 (1997).

75. Borkowski, K. J., Blondin, J. M. \& McCray, R. X-rays from the impact of SN1987A with its circumstellar ring. Ap. J. 477, 281-93 (1997).

76. Blondin, J. M., Wright, E. B., Borkowski, K. J. \& Reynolds, S. P. Transition to the radiative phase in supernova remnants. Ap. J. 500, 342-354 (1998).

77. Vishniac, E. T. The dynamic and gravitational instabilities of spherical shocks. Ap. J. 274, 152-67 (1983).

78. Ryu, D. \& Vishniac, E. T. The Dynamic Instability of Adiabatic Blast Waves. Ap. J 368, 411-25 (1991).

79. Garcia-Segura, G. \& Low, M. M. M. Wolf-Rayet Bubbles. II. Gasdynamical simulations. ApJ 455, 160-174 (1995).

80. Grun, J. et al. Instability of Taylor-Sedov blast waves propagating through a uniform gas. Phys. Rev. Lett. 66, 2738-41 (1991).

81. Laming, J. M. \& Grun, J. Dynamical overstability of radiative blast waves: The atomic physics of shock stability. Phys. Rev. Lett. 89, 125002-125005 (2002).

82. Edwards, M. J. et al. Investigation of ultrafast laser-driven radiative blast waves. Phys. Rev. Lett. 87, 0850041-0850014 (2001).

83. Bozier, J. C. et al. Experimental observation of the radiative wave generated in xenon by a laser-driven supercritical shock. Phys. Rev. Lett. 57, 1304-1307 (1986).

84. Koenig, M. \& al., e. in Shock Compression of Condensed Matter-2001 (ed. al., M. D. F. e.) 1367-1370 (American Institute of Physics, 2001).

85. Budil, K. S. et al. The flexible X-ray imager. Rev. Sci. Inst. 67, 485-8 (1996).

86. Drake, R. P. Equations of state in ionizing and radiating plasmas. Phys. Plasmas, in preparation (2004).

87. Liang, E. \& Keilty, K. An analytic approximation to radiative blast wave evolution. Astrophys. J. 533, 890-892 (2000).

88. Braun, R., Gull, S. F. \& Perley, R. A. Physical process which shapes Cassiopeia A. Nature 257, 395-398 (1987). 
Final Report: Grant Award DE FG52 03 SF22689 (formerly DE FG03 03 SF22689)

89. Herrmann, M. (2003).

90. Leibrandt, D. R. \& Drake, R. P. Validation of the flux-limited diffusion approximation for radiation hydrodynamics. Astrophyscal Journal, submitted (2004). 